\title{
Straight trajectory planning for keyhole neurosurgery in sheep with automatic brain structures segmentation
}

\author{
Alberto Favaro*a, Akash Lad ${ }^{\mathrm{a}}$, Davide Formenti ${ }^{\mathrm{a}}$, Davide Danilo Zani ${ }^{\mathrm{b}}$, Elena De Momi ${ }^{\mathrm{a}}$ \\ ${ }^{a}$ Department of Electronics, Information and Bioengineering, Politecnico di Milano, Milan, Italy \\ ${ }^{b}$ Department of Veterinary Medicine, University of Milan, Milan, Italy
}

\begin{abstract}
In a translational neuroscience/neurosurgery perspective, sheep are considered good candidates to study because of the similarity between their brain and the human one. Automatic planning systems for safe keyhole neurosurgery maximize the probe/catheter distance from vessels and risky structures. This work consists in the development of a trajectories planner for straight catheters placement intended to be used for investigating the drug diffusivity mechanisms in sheep brain.

Automatic brain segmentation of gray matter, white matter and cerebrospinal fluid is achieved using an online available sheep atlas. Ventricles, midbrain and cerebellum segmentation have been also carried out.

The veterinary surgeon is asked to select a target point within the white matter to be reached by the probe and to define an entry area on the brain cortex. To mitigate the risk of hemorrhage during the insertion process, which can prevent the success of the insertion procedure, the trajectory planner performs a curvature analysis of the brain cortex and wipes out from the poll of possible entry points the sulci, as part of brain cortex where superficial blood vessels are naturally located. A limited set of trajectories is then computed and presented to the surgeon, satisfying an optimality criteria based on a cost function which considers the distance from critical brain areas and the whole trajectory length.

The planner proved to be effective in defining rectilinear trajectories accounting for the safety constraints determined by the brain morphology. It also demonstrated a short computational time and good capability in segmenting gyri and sulci surfaces.
\end{abstract}

Keywords: trajectory, planner, sheep, keyhole, neurosurgery, curvature, segmentation, atlas

\section{INTRODUCTION}

Automatic planning systems for safe keyhole neurosurgery have been proposed in literature for electrodes placement in Stereoelectroencephalography [1,2], Deep Brain Stimulation [3] and image-guided neurosurgery [4]. In general, they optimize straight trajectory planning maximizing the distance from vessels and risky structures, such as lateral ventricles, midbrain and cerebellum [1].

The importance of ovine subjects falls within the context of translational neuroscience and neurosurgery, where they are increasingly recognised in several fields as promising candidates for clinical trials with respect to other gyrencephalic species thanks to their greater brain size and body weight [5], a thinner cranial bone, and a similar angioarchitecture of the venous system [6]. Despite the many solutions available in human neurosurgery, to date, none of these has been designed for sheep surgery although the need for a reliable solution is required even in this latter case, not only for ethical issues but also for not prevent the success of the study.

Automatic segmentation procedures give the possibility to highlight anatomical structures of particular concern in the surgical scenario by overtaking those limits characterising manual and semi-automatic procedures, which firstly show high subjectivity (depending upon the user experience) and frequently result to be rather labour-intensive. On the contrary, pure automatic techniques can provide a deterministic tissue labelling capability but, in turn, require a template with the a priori probability that a voxel belongs to a specific structure. Many brain atlases and models are available for humans $[7,8]$ along with software capable to handle them.

The present work proposes a $3 D$ Slicer $\odot$ module (www.slicer.org, hereinafter, Slicer) for trajectory planning able to calculate, accounting for the brain morphology, feasible insertion paths for a linear, rigid catheter intended to be used for

Medical Imaging 2017: Image-Guided Procedures, Robotic Interventions, and Modeling, edited by

Robert J. Webster III, Baowei Fei, Proc. of SPIE Vol. 10135, 101352E · C 2017 SPIE

CCC code: $1605-7422 / 17 / \$ 18 \cdot$ doi: $10.1117 / 12.2254515$

Proc. of SPIE Vol. 10135 101352E-1 
studying cerebral drug diffusion mechanisms. The method aims at laying the foundations for forthcoming more complex planners for flexible catheters in the framework of the EDEN2020 recently funded European project (www.eden2020.eu).

\section{MATERIALS AND METHODS}

The sheep atlas by Nitzsche B. et al. [9] was used for the automatic segmentation procedure: this atlas provides high spatial resolution (isotropic voxel size $0.25 \mathrm{~mm}$ ), features a standard coordinate system according to Talairach space conventions and it is based on averaged $\mathrm{T} 1 \mathrm{w}$ images of 14 Merino sheep ( $\mathrm{T} 1 \mathrm{w}$ incoherent gradient echo, 1.5T, recon matrix 512, slices 110 , voxel size $0.39 \times 0.39 \times 1.0 \mathrm{~mm}$, Gyroscan Interea, Philips). This ensures the conformity with a proper coordinates system, overcomes the drawback arising from inter-individual variability and allows to distinguish white matter (WM), grey matter (GM) and cerebrospinal fluid (CSF) as relevant brain structures. Coupled with the atlas, three intensity-based Tissue Probability Maps (TPMs) are also provided for WM, GM and CSF. They represent the probability that a specific voxel belongs to the afore-mentioned tissue classes.

Our planning method consists in two main steps: a phase consisting in the registration, segmentation and 3D reconstruction, followed by the distance map and straight trajectories computation, as described in the following paragraphs.

\subsection{Registration, Segmentation and 3D reconstruction}

In order to define a suitable trajectory for the catheter, the system requires magnetic resonance (MR) data of the ovine brain. For the scope, one MR dataset was collected from a Bergamasca sheep head (T1w high resolution gradient echo, $0.18 \mathrm{~T}$, recon matrix 512 , slices 23 , voxels size $0.39 \times 0.39 \times 4.4 \mathrm{~mm}$, Esaote, VetMR). MR images undergone a registration process in order to align them with the standardized atlas. This step consisted in finding the affine transformation able to adapt the MR data collected from the sheep so that to fit the atlas dimensions. Registration was performed making use of SPM12 software (www.fil.ion.ucl.ac.uk/spm/software/spm12).

After registering the MR data with the atlas, SPM12 segmentation software was used to segment GM, WM and CSF. By the ModelMaker module available on Slicer, the 3D surfaces of the segmented structures were obtained. Further, a manual segmentation of the lateral, third and fourth ventricles, as well as of cerebellum and midbrain was carried out and approved by a veterinary. These structures will be than considered, in the trajectories computation step, as safety-critical areas to be avoided during the catheter insertion.

\subsubsection{Entry area definition and curvature index computation}

The veterinary surgeon has to manually select the target point within the brain, i.e. the position achieved by the catheter tip, as well as a preferred entry point on the brain cortex. The catheter entry will not be strictly limited to the point selected by the surgeon, rather a circular area of feasible entry points will be provided, which radius can be expanded. Subsequently, in order to reduce the hazard of severe haemorrhage during the catheter placement, which can prevent the success of the procedure, the trajectory planner module wipes out from the poll of possible entry points the sulci, as part of brain where the blood vessels are generally located [10].

The solution adopted makes use of the cortex curvature index to distinct the convex surfaces of the mesh from the concave ones. In doing so, it becomes possible to link with a quantitative index the convex area of the gyri (to which positive index of curvature are associated) and the concave one, which stands for the sulci, that will show negative curvature values. Through a threshold to assign to the such-obtained curvature index it will be so possible to split the gyri from the sulci and, modifying its magnitude, to manipulate the range of gyri surface that will be displayed and consider as feasible entry area.

As the mesh is a non-continuous triangular surface, the afore-mentioned index is computed as a mean curvature, using a discrete approach similar to the ones proposed in [11]. For each vertex $i$ of the mesh, the curvature index $H_{i}$ results from an average, over the number of connected facets $m$, of the curvature value determined by the product between the dihedral angle $\beta_{j}$ and the length of the edge in common between two adjacent facet $e_{j}$, as follows:

$$
H_{i}=\frac{a}{m} \frac{\sum_{j=0}^{m-1}\left\|e_{j}\right\| \beta_{j}}{A}
$$


Where the index $H_{i}$ is normalised with respect to both the area $A$ of the facets considered in the computation and the correction parameter $a$, which accounts for the repetition of the same facet area in the computation. The dihedral angle, instead, $\beta_{j}$ consists in the angle defined by the perpendicular vectors $\vec{n}$ of the two adjacent facets considered, as:

$$
\beta_{i}=\angle\left(\overrightarrow{n_{J-1}}, \overrightarrow{n_{J}}\right)
$$

Such a method is implemented in the VTK library (www.vtk.org), as the filter named vtkcurvature, which demonstrated to be much less computationally expensive if compared to FreeSurfer (surfer.nmr.mgh.harvard.edu), a commonly used software in medical research for the brain, which is here considered as the gold standard for curvature computation: in the experiments, the computational time required for the indexes estimation based on vtkCurvature spanned between a minimum of 2.34 secs and a maximum of 14.29 secs, depending on the mesh resolution, with respect to the time required by FreeSurfer, which is in the order of hours [12]. After the computation of the curvature indexes, the surgeon has the possibility to vary interactively the curvature threshold by a slider implemented in the Slicer module and, in doing so, increase or decrease the gyri surface suitable to be considered as the entry location.

\subsection{Distance map, straight trajectories computation}

After having estimated the curvature values coupled to the cortex surface, all the vertices belonging to the feasible entry area (the one defined by the veterinary surgeon and deprived by the sulci) are considered as candidate entry points for the straight catheter. Then, a rectilinear path is computed from these vertices to the target point.

The implemented cost function $(R)$ associated to each possible trajectory considers, as an input parameter, the Euclidean distance from the safety-critical structures formerly discussed. This consists in linking each voxel of the brain model to the distance from the closest structure. The signed Danielsson distance map filter provided in the ITK library (itk.org) has been used to this scope, providing a value spanning from 0 to 255 (small values for proximity).

Each trajectory has linked a cost function to maximize, which is defined as follows:

$$
R_{i}=\alpha \cdot D_{s t r, i}-\beta \cdot L_{i}
$$

where $D_{s t r, i}$ and $L_{i}$ are the minimum distance from a critical structure along the trajectory and the total trajectory length, whilst $\alpha$ and $\beta$ are two weights, equal to 0.9 and 0.2 , respectively. All those entry points whose trajectory intersects an inner structure or results to be too close to its border $\left(D_{s t r, i}<1.25 \mathrm{~mm}\right)$ are discarded. The ten trajectories achieving the best rank in term of cost function are then proposed to the user.

\section{RESULTS}

\subsection{Automatic segmentation}

The segmentation process, carried out on the registered Bergamasca sheep MR data, produces the results shown in Fig. 1, where WM, GM and CSF are differently coloured.

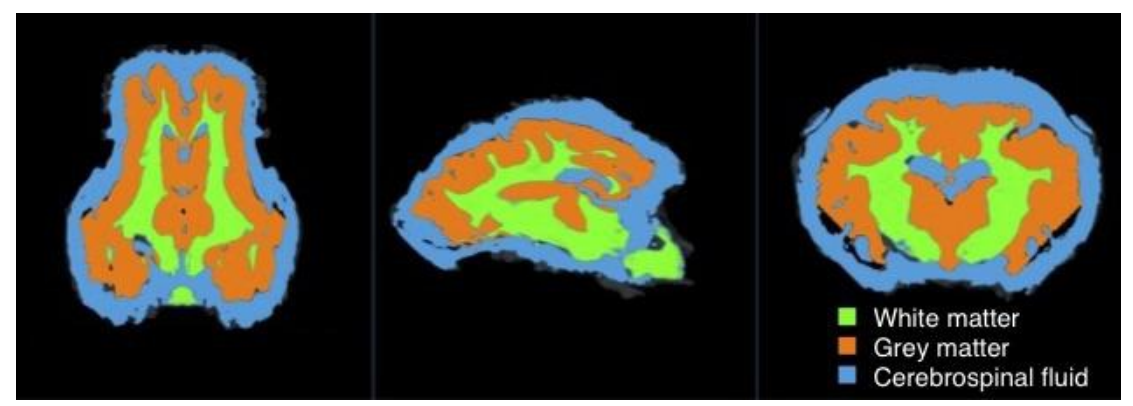

Fig 1. Results of the brain structure segmentation on the Bergamasca sheep MR data using TPMs from the atlas

The correctness of the obtained segmentation was assessed defining a set of 15 axial slices from the original ovine MR data along the coronal section, spanning over the entire brain volume. The Sørensen-Dice coefficient $(Q S)$ was selected 
as the index capable to compare the similarity of the segmented structure generated by the automatic procedure $(X)$ with respect to the ones obtained through a manual way and validated by a veterinary $(Y)$ :

$$
Q S=\frac{2|X \cap Y|}{|X|+|Y|}
$$

Because of the low resolution of the available Bergamasca sheep MR data, a clear discrimination between the grey and white matter was not achievable with the manual procedure, for this reason the comparison was carried out considering the union of the two components. A statistic regarding the obtained DICE values can be evaluated in Tab. 1, where a high value of similarity between automatic and manual approach is shown from the combination of GM and WM. Differently, the median value of the coefficient decreases for the CSF, showing also a higher variability.

Table 1. Variability, minimum and maximum values of the DICE coefficient obtained from the comparison between automatic and manual segmentation procedure for the CSF and the combination of GM and WM.

\begin{tabular}{|c|c|c|c|c|}
\hline Tissue type & Mean [\%] & Std dev [\%] & Min [\%] & Max [\%] \\
\hline CSF & 0.517 & 0.060 & 0.402 & 0.622 \\
\hline GM+WM & 0.937 & 0.026 & 0.869 & 0.965 \\
\hline
\end{tabular}

\subsection{Resizing factor}

For the validation of the proposed method of gyri and sulci segmentation, a comparison with the gold standard was carried out, using a human MR dataset (T1 3D FFE sagittal images, voxel size $0.90 \mathrm{~mm} \times 1.07 \mathrm{~mm} \times 0.90 \mathrm{~mm}$ without any inter-slice gap, then reconstructed and reformatted on the axial plane with $560 \times 560 \times 220$ matrix, $0.45 \mathrm{~mm} \times 0.45$ mm x 0.9 mm, 1.5T, Intera Achieva, Philips Medical System) since FreeSurfer cannot handle sheep brain morphology.

The curvature index obtained via vtkCurvature can be affected by a mesh magnification or reduction, which has the result to expand the curvature range of values a specific vertex can assume by varying the surface dimensions. Different combinations of curvature threshold and reduction factor were iteratively given as input to the vtkCurvature filter when applied to a $15 \mathrm{~mm}$-radius area of cortical surface, looking for the pair which generated the best match with the gold standard in term of recognized gyri area. As a result, a 100-times reduction of the mesh (corresponding to a resizing factor of 0.01), was selected as appropriate for the scope of our work, given that it was able to produce a mismatch of less than $1 \mathrm{~mm}^{2}$.

\subsection{Method validation}

After having defined the best resizing value for the vtkCurvature algorithm, its gyri and sulci recognition capability was compared with the gold standard on a set of ten different areas of $15-\mathrm{mm}$ radius, defined on the human MR cortical surface, as shown in Fig. 2.

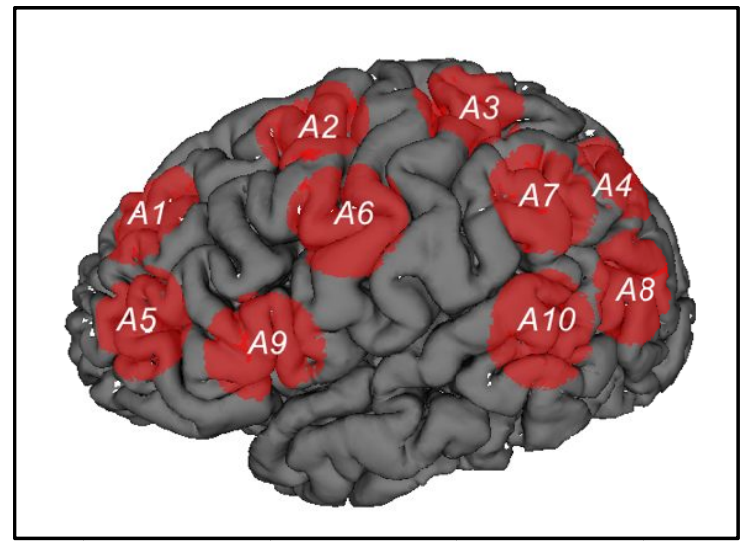

Fig 2. Set of 15-mm circular areas defined on the human cortical brain. 
Each area undergone a gyri and sulci segmentation by means of the goal standard approach at three levels of curvature threshold, resulting in a set of thirty differently-segmented configurations. It is important to notice that Freesurfer considers negative values of curvature for convex surfaces (gyri) and positive values for the concave ones (sulci), therefore its convention is the opposite with respect to the one implemented in vtkCurvature. The values of curvature threshold selected from the outcome produced by FreeSurfer were the following: THR $1=0 \mathrm{~mm}^{-1}$, representing an ideal equal separation between gyri and sulci, THR $2=0.1 \mathrm{~mm}^{-1}$, which selects a larger gyri surface, THR $3=-0.1 \mathrm{~mm}^{-1}$, a more conservative value having the effect of reducing the accessible gyri surface. The method based on vtkCurvature was also applied, and the mismatch between the gyri surface recognized by the two approaches was computed, resulting in the outcome presented in Fig. 3.

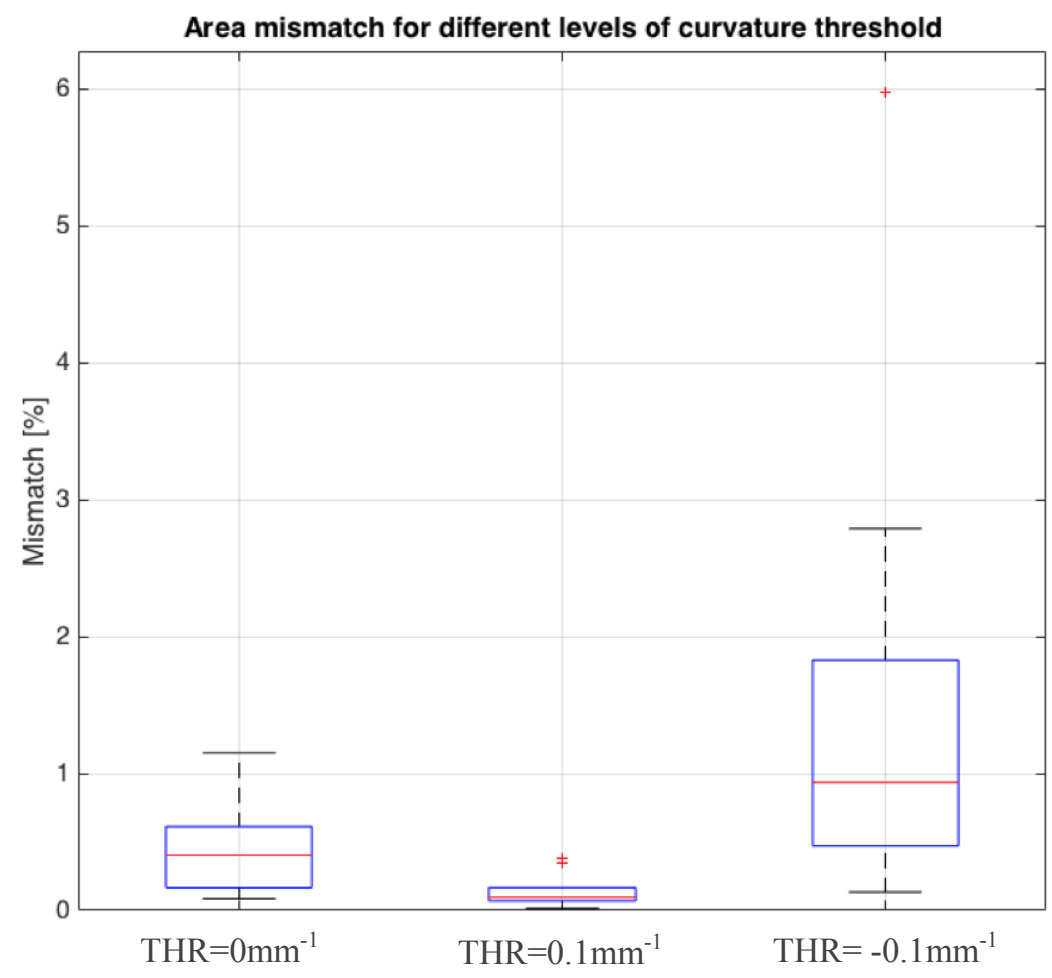

Fig 3. Variability of the area mismatch in the set of ten curvilinear cortical surfaces for the three different threshold configurations.

In particular, it can be noticed that, for the second threshold value, where a larger gyri surface was considered, a very good agreement with the gold standard was obtained, while the degree of mismatch is higher for the first and third values, where lower threshold levels were selected on the gold standard, corresponding to less gyri surface. In Fig. 4, the best results in term of area matching are displayed for the three levels of curvature threshold cited above in the case the occipital area A8 is considered. 


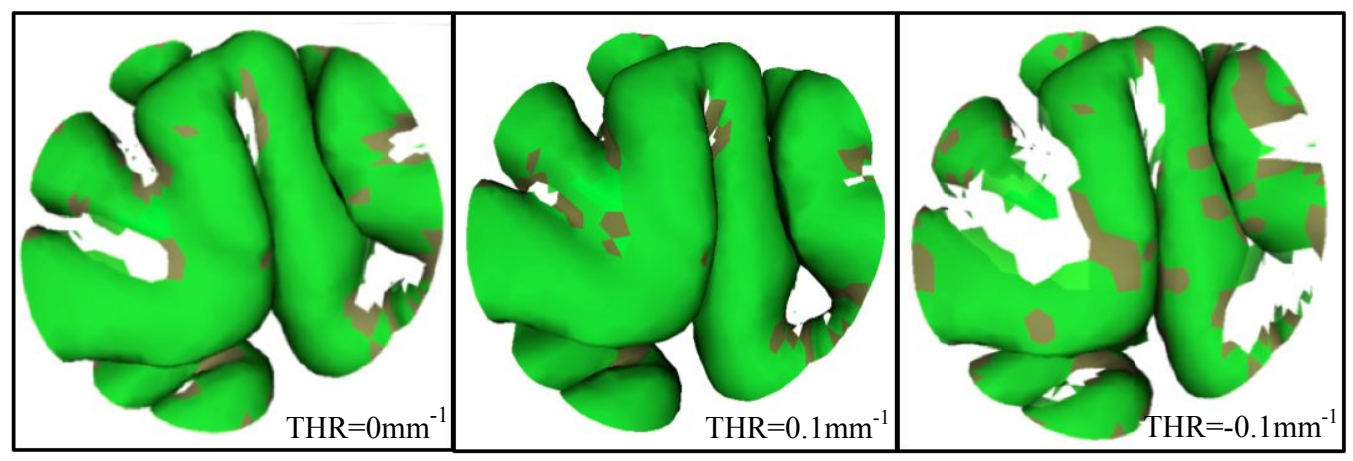

Fig 4. Representation of the lowest mismatch condition for the different threshold values between the vtkCurvature (green) solution and the gold standard (brown) computed for the occipital area A8.

\subsection{Trajectories definition}

Because of the too coarse resolution of the available MR dataset from the Bergamasca sheep, the proposed vtkCurvature method was tested directly on the ovine brain atlas by Nitzsche B. et al. To do so, the Trajectory Planning module was used, which was specifically developed for this purpose. In Fig. 5 the two lateral ventricles, as well as the midbrain and cerebellum resulting from the manual registration are highlighted in different colors. On the brain cortex, the result of the segmentation process is shown in the circular entry area defined by the user in accordance with the desired curvature threshold. The entry and target points are also drawn, as well as the three straight trajectories connecting the entry area to the target point, where the green-colored one represents the path linked to the highest value assumed by the cost function, the red the one coupled to the lowest and the yellow the intermediate one.

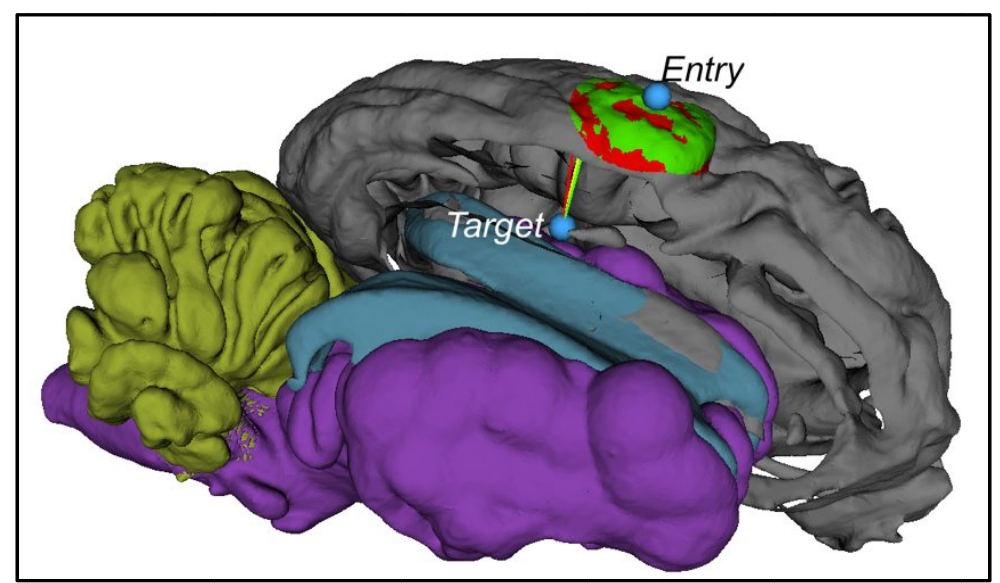

Fig 5. Result of trajectories estimation based on the entry area and the target point definition. In gray the cortex, where gyri and sulci are presented in green and reed respectively. In cyan the two ventricles, in purple the midbrain, in olive the cerebellum.

\section{CONCLUSIONS}

To date, sheep are increasingly recognised in translational neuroscience/neurosurgery as promising candidates for neurological clinical trials thanks to the many similarities with the human brain. According to the authors knowledge, no other planner similar to the one herein proposed has been developed for ovine samples.

The main purpose of the work was to develop, as a Slicer module, a trajectory planner customized for sheep brain, whose workflow embraces also the automatic segmentation of relevant brain areas, by exploiting open-source atlas available on the web, as well as some manually segmented critical structures. The system allows to define a set of rectilinear 
trajectories feasible for straight probe or catheter insertion in ovine brain surgery after having defined the target point and the entry area.

By looking at the results, a good automatic segmentation capability was highlighted in the case of WM and GM, even though the coarse resolution of the original Bergamasca sheep MR data required to implement a solution based on the combination of the two matters, rather than to assess them individually. In the case of the CSF, instead, the outcome results worse but, also in this case, the image quality has probably contributed in biasing the result.

The proposed solution, based on vtkCurvature filter, shown a good matching capability when compared to FreeSurfer, which was considered as gold standard, at three different threshold configurations, although the comparison demonstrated that the outcome worsened when the threshold tended to be more conservative and the gyri surface reduced. In addition to this, the method here proposed needs some refinements, since it tends to generate some holes in the gyri surface segmentation, especially when implemented on low resolution MR data and jagged surfaces. The problem can be probably tackle through a smoothing phase to be carried out before the curvature computation. For the scope, the best combination of smoothing and resizing factors will be investigated in a future work.

The possibility to use a higher resolved ovine dataset will give the possibility to better assess the segmentation property of the TPSs, as well as to develop an automatic segmentation solution also for the critical areas herein considered. Further, the introduction of the brain angiostructure as well as Diffusion Tensor Imaging (DTI) data would represent a relevant extra constraint for the planning phase, which should also embrace the capability of generating curvilinear trajectories for steerable catheter insertion.

\section{AKNOWLEDGEMENTS}

This project has received funding from the European Union's Horizon 2020 research and innovation program under grant agreement No 688279.

This work is not being and has not been submitted for publication or presentation elsewhere.

\section{REFERENCES}

[1] De Momi, E., Caborni, C., Cardinale, F., Casaceli, G., Castana, L., Cossu, M., et al. (2014). Multi-trajectories automatic planner for StereoElectroEncephaloGraphy (SEEG). International Journal of Computer Assisted Radiology and Surgery, 9(6), 1087-1097.

[2] De Momi, E., Caborni, C., Cardinale, F., Castana, L., Casaceli, G., Cossu, M., et al. (2013). Automatic trajectory planner for StereoElectroEncephaloGraphy procedures: A retrospective study. IEEE Transactions on Bio-Medical Engineering, 60(4), 986-993.

[3] Essert, C., Fernandez-Vidal, S., Capobianco, A., Haegelen, C., Karachi, C., Bardinet, E., et al. (2015). Statistical study of parameters for deep brain stimulation automatic preoperative planning of electrodes trajectories. International Journal of Computer Assisted Radiology and Surgery, 10(12), 1973-1983.

[4] Shamir, R. R., Tamir, I., Dabool, E., Joskowicz, L., \& Shoshan, Y. (2010). A method for planning safe trajectories in image-guided keyhole neurosurgery. Medical Image Computing and Computer-Assisted Intervention : MICCAI International Conference on Medical Image Computing and Computer-Assisted Intervention, 13( $\mathrm{Pt} 3)$, 457-464.

[5] Forschler, A., Boltze, J., Waldmin, D., Gille, U., \& Zimmer, C. (2007). MRI of experimental focal cerebral ischemia in sheep. [MR-Bildgebung eines experimentellen Schlaganfallmodells beim Schaf] RoFo : Fortschritte Auf Dem Gebiete Der Rontgenstrahlen Und Der Nuklearmedizin, 179(5), 516-524.

[6] Hoffmann, A., Stoffel, M. H., Nitzsche, B., Lobsien, D., Seeger, J., Schneider, H., et al. (2014). The ovine cerebral venous system: Comparative anatomy, visualization, and implications for translational research. PloS One, 9(4), e92990.

[7] Van der Lijn, F., de Bruijne, M., Klein, S., den Heijer, T., Hoogendam, Y. Y., van der Lugt, A., et al. (2012). Automated brain structure segmentation based on atlas registration and appearance models. IEEE Transactions 
on Medical Imaging, 31(2), 276-286.

[8] Tu, Z., Narr, K. L., Dollar, P., Dinov, I., Thompson, P. M., \& Toga, A. W. (2008). Brain anatomical structure segmentation by hybrid discriminative/generative models. IEEE Transactions on Medical Imaging, 27(4), 495508.

[9] Nitzsche, B., Frey, S., Collins, L. D., Seeger, J., Lobsien, D., Dreyer, A., et al. (2015). A stereotaxic, population-averaged $\mathrm{T} 1 \mathrm{w}$ ovine brain atlas including cerebral morphology and tissue volumes. Frontiers in Neuroanatomy, 9, 69.

[10] Viviani, R. (2016). A digital atlas of middle to large brain vessels and their relation to cortical and subcortical structures. Frontiers in Neuroanatomy, 10

[11] Dyn, Nira, et al. "Optimizing 3D triangulations using discrete curvature analysis." Mathematical methods for curves and surfaces 38.8 (2001): 135-146.

[12] Delgado, J., et al. "Improving the Execution Performance of FreeSurfer." Neuroinformatics 12.3 (2014): $413-$ 421. 